\title{
Old Unreduced Elbow Fracture and Fracture Dislocation: Treatment With Open Reduction and Hinge External Fixation
}

\author{
Reza Shahryar Kamrani ${ }^{1}$; Leili Farhadi ${ }^{1}$; Leila Oryadi Zanjani ${ }^{1, *}$ \\ ${ }^{1}$ Department of Orthopedic and Trauma Surgery, Shariati Hospital, Tehran University of Medical Sciences, Tehran, IR Iran \\ ${ }^{*}$ Corresponding author: Leila Oryadi Zanjani, Department of Orthopedic and Trauma Surgery, Shariati Hospital, Tehran University of Medical Sciences, Tehran, IR Iran. Tel: \\ +98-9121017913, Fax:+98-2188220055, E-mail: leila_zanjani@yahoo.com
}

Received: January 27, 2015; Revised: February 21, 2015; Accepted: March 11, 2015

\begin{abstract}
Background: Old unreduced elbow dislocation and fracture-dislocation, although rare, are one of the challenges in orthopedic surgery. The application of the hinged elbow external fixator can confer early range of motion after open reduction and reconstruction in spite of severe soft tissue and bony injury.

Objectives: This study presents our results of open reduction and reconstruction in old simple elbow dislocation and old elbow fracturedislocation.

Patients and Methods: In 12 patients with old elbow dislocation, open reduction and bone and soft tissue reconstruction was done and early postoperative range of motion was begun with the aid of hinged elbow external fixators.

Results: The mean elbow range of motion at the final follow-up was 98 degrees $(\mathrm{SD}=10)$. According to the Mayo Elbow Performance Index score, 6 patients achieved excellent results and 2 other had good results with a mean value of $92(\mathrm{SD}=8)$.

Conclusions: We recommend hinged elbow external fixation in old elbow dislocation for early range of motion and maintenance of concentric reduction with acceptable results.
\end{abstract}

Keywords: Dislocation; Elbow; External fixators

\section{Background}

Acute elbow dislocation is the second most common major joint dislocation in adults (1). This dislocation is usually treated with closed reduction. Old unreduced elbow dislocation is rare in developed countries but may occur in multiple injured patients, head trauma, or when the patient does not seek medical treatment. In contrast to acute dislocation, old elbow dislocation and fracturedislocation management is difficult with suboptimal results. The traditional treatment of old elbow dislocation and fracture-dislocation consists of open reduction, bone structures reconstruction with or without collateral ligaments reconstruction, and temporary arthrodesis (1). With the development of hinge external fixators, early range of motion (ROM) can be started along with the maintenance of joint reduction and reconstruction of the bone and soft tissue structures $(2,3)$. This technique has been shown to improve the results of the treatment in old unreduced elbow dislocation and fracturedislocation. Because of the rare incidence in developed countries, there are few studies with small numbers of cases that focus on the treatment of these injuries with early ROM (1). So the optimal treatment strategies like the surgical approach and the importance of the bony and soft tissue reconstruction are not clear.

\section{Objectives}

We reviewed the results of open reduction and hinge external fixator application in our patients with elbow dislocation or fracture-dislocation treated one month or more after their initial injury. The results were compared with other similar studies.

\section{Patients and Methods}

\subsection{Patients}

In this study, 12 patients, treated for old unreduced elbow fracture or fracture-dislocation were evaluated. Only patients with more than one month's delay in treatment and complete ulnohumeral dislocation with at least a one-year follow-up period were included. Patients with subluxation or recurrent dislocation were excluded. This retrospective study was approved by our institutional review board.

\subsection{Operative Technique}

Under general anesthesia with the patient in the semilateral position and through a longitudinal posterior skin incision, the ulnar nerve was exposed, released, and prepared for anterior transposition at the end of the pro- 
cedure. With preoperative planning, decision was made as to do either olecranon osteotomy or paratricipital approach. We preferred to do olecranon osteotomy when there was huge heterotopic ossification or intra-articular fracture or when obstacles made it difficult to obtain a concentric reduction (Figure 1). In these situations, better exposure with olecranon osteotomy helped us to minimize soft tissue detachment from the ulnoradial component. Otherwise, we would choose the paratriceps approach. Then, the soft tissue was released as a sleeve from the distal humerus, including any remnant of the collateral ligaments. All fibrous tissue was removed from the joint space. If there was any displaced fracture interfering with stability, it was reduced or reconstructed and fixed; otherwise, it was removed. Thereafter, the ulnohumeral joint was reduced and ROM was evaluated. In the olecranon osteotomy technique, the olecranon was first fixed temporarily with a towel clip and then ROM was evaluated. In the long standing cases, usually there was a limitation of flexion. In these cases, with a fractional lengthening through the musculotendinous junction of the distal triceps (Figure 2) and manipulation, the desired
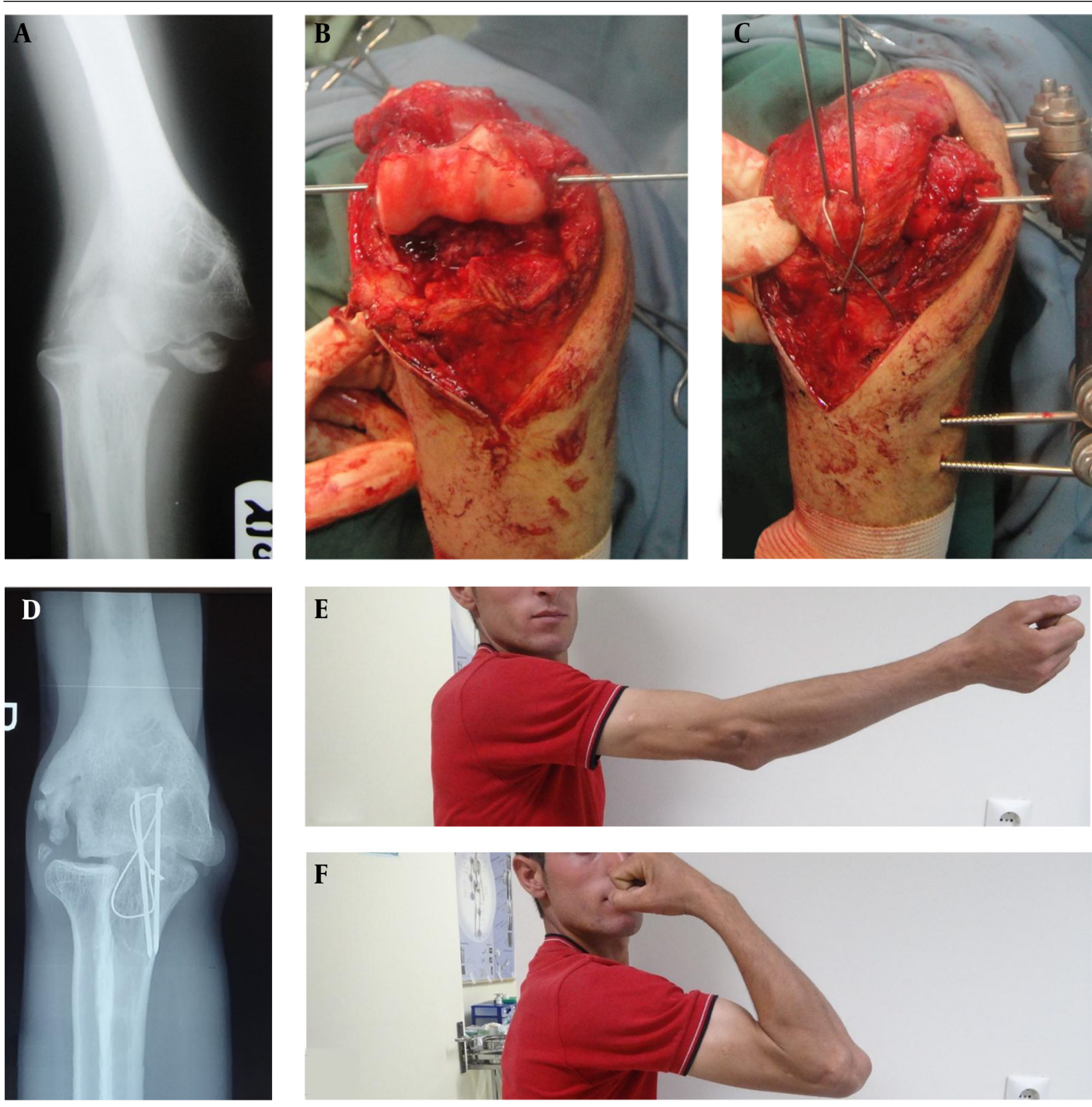

Figure 1. A, Preoperative X-Ray; B, Articular Surface Exposure with Olecranon Osteotomy; C, Tension Band Fixation and Hinge External Fixator Application; D, Final X-Ray after External Fixator Removal; E, F, Postoperative Range of Motion 
Shahryar Kamrani R et al.

ROM was achieved. Subsequently, the joint was redislocated. The hinge external fixator's guide pin was inserted under direct view, parallel to the inferior border and the anterior border of the trochlea from the anteroinferior corner of the medial epicondyle to the anteroinferior corner of the lateral epicondyle. The joint was reduced, and the olecranon was fixed with the tension band wiring technique in the cases of olecranon osteotomies (Figure $1 \mathrm{C}$ ). The shanzes of the proximal and distal arms of the external fixator were inserted in the distal humerus under direct vision and in the proximal ulna percutaneously. In our first 4 patients, we used the Elbow Hinge Fixator (Synthes West Chester, PA) and in the other patients the Dynamic Joint Distractor II (Stryker Orthopaedics, Mahwah, NJ). Joint ROM was evaluated again for smooth and congruent motion. The guide pin was removed, and the medial and lateral soft tissue was repaired without trying for the isolation and reattachment of individual collateral ligaments. The ulnar nerve was transposed to anterior subcutaneously. The patients were advised to begin elbow motion the day after surgery. The fixator was removed after 6 to 8 weeks. The patients were visited at $3,6,9$, and 12 months after surgery. They were recalled for visit for the last follow-up if their last visit was longer than 6 months. Any complication intra operatively or at follow-up was recorded.

\subsection{Assessments}

In preoperative visit time between injury and treatment, previous treatment, fractures around the elbow and concomitant injuries, and elbow ROM were recorded. Elbow anteroposterior and lateral view X-ray and computed tomography (CT) scan were obtained for operative planning. At the last follow-up, we evaluated the final elbow ROM, elbow stability, and Mayo Elbow Performance Index score. Final radiographs also were evaluated for concentric reduction and heterotopic ossification.

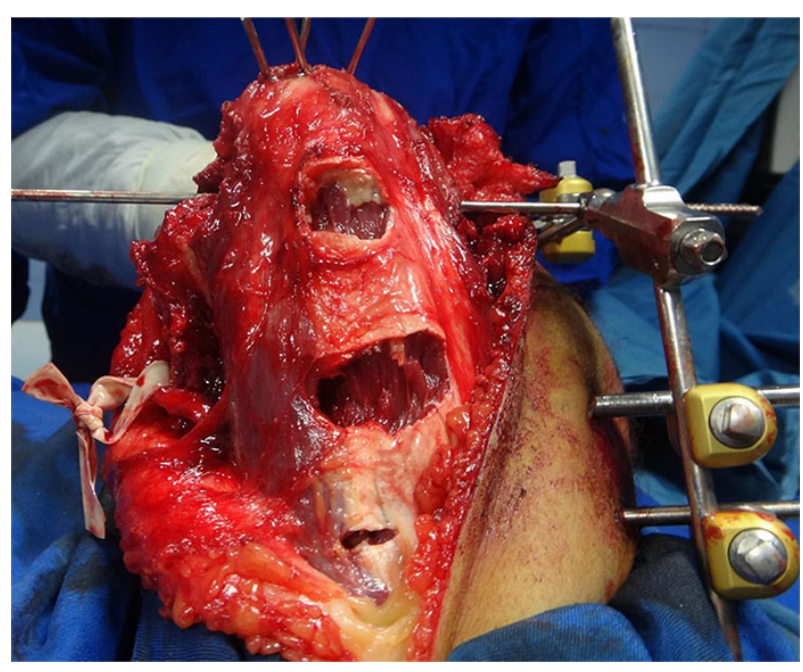

Figure 2. Triceps Fractional Lengthening

\subsection{Statistical Analysis}

There were 9 patients with dislocation and 3 patients with fracture-dislocation. Even though we gathered all the results, for comparison with other similar studies, we only performed statistical analysis in patients with isolated dislocation in terms of ROM and Mayo Elbow Performance Index score.

The data are presented as mean and standard deviation (SD). The paired samples T test was used to compare the continuous variables. A P $<0.05$ was considered statistically significant.

\section{Results}

Between August 2008 and February 2013, we treated 12 patients who met the inclusion criteria of this study. The patients included 11 males and one female (Patient 2) at a mean age of 32 years (range $=15-48$ ). Five patients had been treated previously by orthopedic surgeons before their referral to us: 2 cases of terrible triad, treated with open reduction and radial head excision, and 3 cases of simple dislocation, treated with closed reduction. Concomitant injuries were extensive soft tissue injuries with both bone fracture of the ipsilateral forearm (Patient 6) and head trauma requiring Intensive Care Unit admission for 2 months (Patient 2). There were 3 patients with fracture-dislocation, 2 patients with terrible triad, and one patient with lateral condylar fracture. In these 3 patients, bone reconstructions were done with different techniques. In one patient with terrible triad, reattachment of the anterior capsule (Patient 8) was done. The other case of terrible triad (Patient 9) underwent reconstruction of the coronoid with the tip of the olecranon. Neither of them needed radial head replacement. The last one (Patient 10) was treated with lateral condyle open reduction and internal fixation. The details are summarized in Table 1.

At the final follow-up, Patient 6 had complete stiffness of the elbow, which we will discuss later. With the exclusion of this patient, in the other 8 patients with pure elbow dislocation, the mean elbow ROM at the final visit was 98 degrees $(S D=10)$, which was significantly higher than the preoperative ROM with a mean value of 18 degrees $(\mathrm{SD}=$ 17) $(\mathrm{P}=0.000)$. According to the Mayo Elbow Performance Index score, 6 patients achieved excellent results and 2 other had good results with a mean value of $92(\mathrm{SD}=8)$. All the patients showed concentric ulnohumeral reduction at the last follow-up.

We did olecranon osteotomy in 5 patients. The postoperative treatment was the same as that in the patients without osteotomy. In all of these patients, olecranon osteotomy healed without problem. In one patient, it showed union at 6 months and in the others it healed at 3 months.

We had 3 major complications in our patients. In one patient (Patient 2), we detected radial head dislocation after surgery and she underwent radial head excision 6 
months after index injury. In one patient (Patient 6), we faced complete stiffness of the elbow and later exploration showed an unsalvageable joint surface. In our opinion, this problem is due to primary joint surface dam- age (Figure 3) rather than the treatment. Finally, in one patient (Patient 10), lateral condyle non-union failed to heal and a second operation was done with vascularized bone graft.
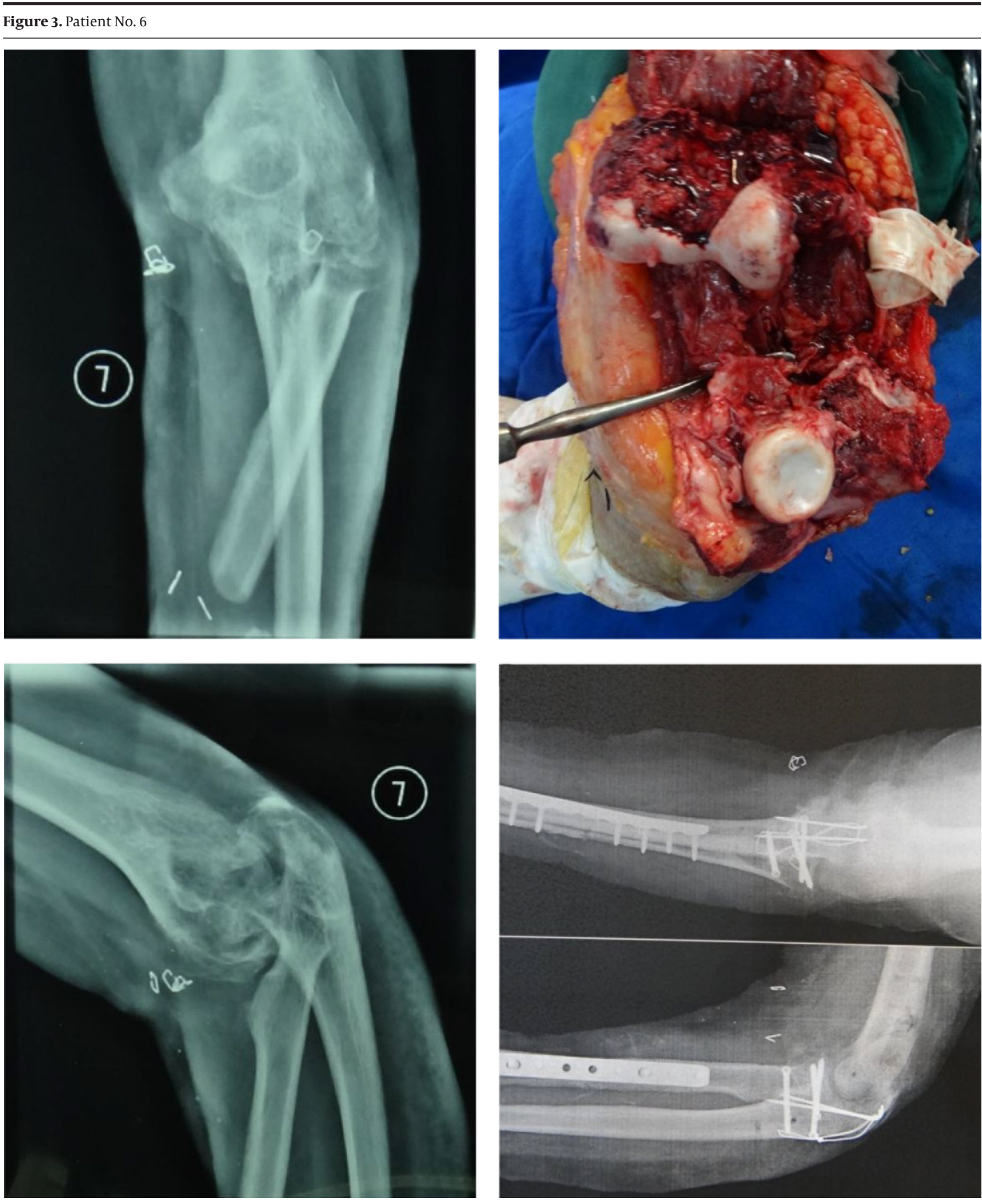

A, Preoperative X-Ray; B, Articular Surface Damage; C, Postoperative X-Ray 
Shahryar Kamrani R et al.

Table 1. Detailed Data of Patients with Old Elbow-Joint Dislocation or Fracture-Dislocation ${ }^{\text {a }}$

\begin{tabular}{|c|c|c|c|c|c|c|c|c|c|c|c|}
\hline No. & Age & Prev Tx & Type & $\begin{array}{c}\text { Concomitant } \\
\text { Injury }\end{array}$ & $\begin{array}{c}\text { Delay of } \\
\text { Treatment, mo }\end{array}$ & $\begin{array}{c}\text { Olec } \\
\text { Osteot }\end{array}$ & $\begin{array}{c}\text { Preop } \\
\text { ROM }\end{array}$ & $\begin{array}{c}\text { Last FU } \\
\text { ROM }\end{array}$ & $\begin{array}{l}\text { Mayo } \\
\text { score }\end{array}$ & $\begin{array}{c}\text { FU time, } \\
\text { mo }\end{array}$ & Complication \\
\hline 1 & 18 & No & Dislocation & & 2 & + & $45-60$ & $25-130$ & 100 & 51 & \\
\hline 2 & 28 & $\mathrm{CR}$ & Dislocation & Head trauma & 4 & - & $20-30$ & $30-130$ & 90 & 28 & Radial head Dx \\
\hline 3 & 19 & No & Dislocation & & 4 & - & $55-70$ & $30-120$ & 80 & 12 & \\
\hline 4 & 15 & No & Dislocation & & 3 & + & $30-90$ & $30-120$ & 95 & 24 & \\
\hline 5 & 48 & $\mathrm{CR}$ & Dislocation & & 2 & - & $45-60$ & $30-130$ & 95 & 12 & \\
\hline 6 & 45 & No & Dislocation & $\begin{array}{l}\text { Forearm soft } \\
\text { tissue }\end{array}$ & 4 & + & $70-75$ & $90-90$ & - & 35 & Elbow ankylosis \\
\hline 7 & 26 & No & Dislocation & & 12 & + & $0-5$ & $10-100$ & 80 & 12 & \\
\hline 8 & 40 & $\begin{array}{l}\text { OR, RH } \\
\text { Ex, PCP }\end{array}$ & $\begin{array}{c}\text { Terrible } \\
\text { triad }\end{array}$ & & 2 & - & $45-65$ & $25-130$ & - & 46 & \\
\hline 9 & 36 & $\begin{array}{c}\text { OR, RH } \\
\text { Ex, Cr Fix }\end{array}$ & $\begin{array}{c}\text { Terrible } \\
\text { triad }\end{array}$ & $\begin{array}{l}\text { Ipsilateral } \\
\text { Ulna Fx }\end{array}$ & 2 & - & $40-70$ & $20-100$ & - & 12 & \\
\hline 10 & 28 & No & $\begin{array}{c}\text { Lat condyle } \\
\text { Fx }\end{array}$ & & 12 & + & $0-10$ & $45-120$ & - & 12 & Lat. condyle NU \\
\hline 11 & 46 & No & Dislocation & & 3 & - & $10-20$ & $20-110$ & 95 & 12 & $\begin{array}{l}\text { Heterotopic } \\
\text { ossification }\end{array}$ \\
\hline 12 & 35 & CR & Dislocation & & 1 & - & $30-45$ & $0-120$ & 100 & 12 & \\
\hline
\end{tabular}

\section{Discussion}

Elbow dislocation is a common dislocation among the major joints in adults with usually simple and satisfying treatment (1). In contrast, old elbow dislocation is rare with difficult treatment. Old elbow dislocation is more common in developing countries $(4,5)$. In these countries, it is difficult to use new technology such as the hinge external fixator because of the prohibitive costs. On the other hand in the developed countries, this neglected dislocation is rare. Consequently, there is a dearth of data on the results of its treatment with early ROM using a hinge external fixator (1). The best approach to old unreduced elbow fracture and fracture dislocation is, therefore, still unclear.

Almost all authors prefer to use the posterior approach for open reduction, while Potini et al. (6) prefer medial over the top, Mahaisavariya et al. (4) opt for triceps splitting, and Coulibaly et al. (5) and Jupiter (2) favor the paratriceps approach. We used either paratriceps or transolecranon approach with the same encouraging results. We did not make any change to our postoperative protocol because of olecranon osteotomy, and nor did we face any additional complications related to osteotomy. What is more, we enjoyed a better view of the joint structures. Reconstruction of the joint was also simpler in this approach. We would not suggest the application of olecranon osteotomy in all cases; nevertheless, we never tend to hesitate to utilize this approach in difficult cases.

Triceps contraction is a problem, especially in long-term neglected dislocations $(4,5)$. Triceps lengthening is mandatory in such cases. The most common type of lengthening is the V-Y plasty of the triceps. We were forced to lengthen the triceps in 3 of our patients with 8,12 , and 12 months' delay in treatment, respectively. We performed a fractional lengthening of the triceps in the musculotendinous portion and manipulation with good results. The number of cases is too small to formulate a suggestion, but it could be considered as an option.

There are some controversies about the importance of the repair or reconstruction of the collateral ligaments with the application of the hinge external fixator and resumption of the elbow motion very soon after surgery (2, 4-6). Our study supports the opinion that repair or reconstruction of the collateral ligaments is not mandatory for a concentric reduction and stable joint at the final followup in this treatment technique.

Our English literature review of studies with at least 5 patients with old dislocation and fracture-dislocation treated with hinge external fixation and early ROM yielded 4 papers. Ruch and Triepel (3) reported 8 patients treated with the external fixator: 5 of them in old elbow fracture-dislocation. They had an average of 84-degree elbow ROM with an average flexion contraction of 33 degrees, and all the patients showed concentric reduction at the final follow-up. Ring et al. (7) reviewed 13 patients with old unreduced elbow fracture-dislocation: all the patients recovered elbow stability with an average Mayo 
Elbow Performance Index score of 84 and average elbow motion of 99 degrees. Potini et al. (6) retrospectively reviewed 7 patients with old elbow fracture-dislocation with at least one month's delay in treatment. At least 5 of their patients had fractures interfering with elbow stability. The average elbow ROM reached 120 degrees with stable joint in their patients. The authors confronted major complications in 4 of their patients. None of these papers could be compared with our study, which is mainly focused on chronic dislocations. Jupiter and Ring (2) published the largest case-series with old elbow dislocation treated via open reduction and hinge external fixators in 5 patients with old elbow dislocation without associated fracture who required no lengthening of the triceps or other soft tissues. The investigators also used a passive gear incorporated into the hinge external fixator and drew upon gradual active mobilization as their postoperative treatment. Their study population had an average age of 49 years, and all the patients had medical attention after their injury. The delay in treatment was an average of 11 weeks. The authors found stable concentric reduction in all of their patients at the last follow-up. The average arc of elbow motion was 123 degrees with 136-degree flexion and 13-degree flexion contraction. The average of the Mayo Elbow Performance Index score was 89. The results of elbow ROM and Mayo score were superior in Jupiter and Ring's study. However, there are some differences between their study and ours. The delay to surgical treatment is significantly longer in our series, obliging us to do fractional lengthening in 2 of our 8 patients. Another point of great significance is the efficacy of physical therapy after treatment. Most of our patients came from provinces far from the capital city, which precluded us from providing them with physical therapy under our own supervision. We merely trained them and their family regarding active-assisted early ROM. Our results, therefore, may have been affected by these factors. Be that as it may, all of our patients gained a stable painless elbow with functional ROM.

First and foremost among the limitations of the present study is its low sample volume. Nonetheless, it is still one of the largest studies in the field of treatment of elbow dislocation and fracture-dislocation with the application of the hinge external fixator. Furthermore, to the best of our knowledge, it is also the largest case-series of old elbow dislocation treated with this technique $(1,2)$. Report- ing old dislocation together with old fracture-dislocation is another weak point of our study, but it is deserving of note that it is not uncommon in other studies (4-6). To our mind, these two categories share the same principles of treatment. We also sought the objective of evaluating the role of olecranon osteotomy in the treatment with hinge elbow fixation. Finally, we assessed the results of pure old dislocations separately for comparison with other studies. We excluded one of our patients from statistical evaluation (Patient 6). As was mentioned before, we are of the opinion that the complication of this patient, who lost his elbow motion, was due to primary articular surface damage and, as such, cannot be considered a problem related to the treatment. Although we could not compare patients with and without olecranon osteotomy, we have been able to introduce this option for difficult cases. The present study is retrospective in its design; however, in our view in such a rare condition, this can offer us a better concept of treatment options.

\section{Authors' Contributions}

Study concept and design: Reza Shahryar Kamrani and Leili Farhadi; Analysis and interpretation of data: Leila Oryadi Zanjani; Drafting of the manuscript: Reza Shahryar Kamrani; Critical revision of the manuscript for important intellectual content: Reza Shahryar Kamrani, Leili Farhadi; Statistical analysis: Leila Oryadi Zanjani; Study supervision: Reza Shahryar Kamrani.

\section{References}

1. Ivo R, Mader K, Dargel J, Pennig D. Treatment of chronically unreduced complex dislocations of the elbow. Strategies Trauma Limb Reconstr. 2009;4(2):49-55.

2. Jupiter JB, Ring D. Treatment of unreduced elbow dislocations with hinged external fixation. J Bone Joint Surg Am. 2002;84A(9):1630-5.

3. Ruch DS, Triepel CR. Hinged elbow fixation for recurrent instability following fracture dislocation. Injury. 2001;32 Suppl 4:SD70-8.

4. Mahaisavariya B, Laupattarakasem W. Neglected dislocation of the elbow. Clin Orthop Relat Res. 2005(431):21-5.

5. Coulibaly NF, Tiemdjo H, Sane AD, Sarr YF, Ndiaye A, Seye S. Posterior approach for surgical treatment of neglected elbow dislocation. Orthop Traumatol Surg Res. 2012;98(5):552-8.

6. Potini VC, Ogunro S, Henry PD, Ahmed I, Tan V. Complications associated with hinged external fixation for chronic elbow dislocations. J Hand Surg Am. 2015;40(4):730-7.

7. Ring D, Hannouche D, Jupiter JB. Surgical treatment of persistent dislocation or subluxation of the ulnohumeral joint after fracture-dislocation of the elbow.J Hand Surg Am. 2004;29(3):470-80. 